\title{
Molar incisor malformation in six cases: Description and diagnostic
}

\section{protocol}

Ingvild Johnsen Brusevold , DDS PhD ","; Thea Martine Granvoll Bie, DDS*; Christine Sophie Baumgartner, DDS, ${ }^{*}$ Runa Das, DDS ${ }^{+}$I Ivar Espelid, DDS, PhD*

${ }^{*}$ Department of Paediatric Dentistry and Behavioural Science, Institute of Clinical Dentistry, Faculty of Dentistry,

University of Oslo

"Department of Oral Biology, Faculty of Dentistry,

University of Oslo

+ Oral Health Centre of Expertise in Mid-Norway, 7030 Trondheim

Correspondence to:

Ingvild Johnsen Brusevold

*Department of Pediatric Dentistry and Behavioural Science, Institute of Clinical Dentistry, University of Oslo, Box 1109, Blindern, 0317 Oslo, Norway

e-mail: i.j.brusevold@odont.uio.no

phone: 004722852158

Word count: Abstract: 195, Whole manuscript: 4826. References: 25 Figures: 8. Tables: 1

Funding: University of Oslo, Norway and grants from Tannlegeundervisningens fond and Norske Tannlegers fond til Tannlegevitenskapens fremme.

Conflicts of interest: None 


\title{
Statement of clinical relevance
}

Molar incisor malformation (MIM) is a newly recognised condition. The dental team should be aware of this condition to make the correct diagnosis and make a control and treatment plan to avoid infection, pain and early tooth loss in affected children.

\begin{abstract}
Objective: The term molar incisor malformation (MIM) has recently been presented in scientific literature, where it is described as a condition with localized impaired root development. We here present six recently discovered cases referred to our departments.
\end{abstract}

Study design: The patients were enrolled in the study after referral and examined clinically and radiologically. Two extracted teeth were further examined with micro-CT or microscopy.

Results: Affected teeth were first permanent molars with hypoplastic roots, narrow pulp chambers and a hypercalcified dentine layer cervical to the pulp chamber. Two of the cases also had cervical constrictions on the upper incisors. They were aged 8-12 years, presently healthy, but had experienced serious medical conditions located to the head and neck region in their first year of life. Some of the cases had been referred because of acute infections and pain.

Conclusion: In five out of six patients severe health problems early in life located in the head and neck region may be associated with root malformation in molars and incisors. Patients with MIM need to be followed closely and extractions should be planned at the right time to avoid unnecessary infections and pain in addition to orthodontic problems. 


\section{Introduction}

The formation of permanent first molars and incisors starts around the time of birth, and serious medical insults to the child during the first two-three years of life may affect tooth development. The occurrence of molar incisor hypomineralization $(\mathrm{MIH})$ is a well-known, but poorly understood, condition affecting the mineralization of permanent first molars and incisors of $2.4-40 \%$ of individuals according to prevalence worldwide studies ${ }^{1}$. Recently, a new condition has been described affecting the roots of the same teeth. So far, only six publications, all published within the last two years, describe the condition ${ }^{2-7}$. In these publications, the terms "root malformation", "molar incisor malformation (MIM)" and "molar root-incisor malformation (MRIM)" have all been used to describe the phenomenon.

From these publications, the condition is characterized by disturbances in root development of all first permanent molars. The roots are very slender, malformed and sometimes absent. In some patients, there are constrictions located to the cervical part of the crown of the permanent incisors. There are also cases where the roots of the second primary molars are affected.

Tooth development is tightly genetically controlled, starting from thickening of the epithelium to form the dental lamina ${ }^{8}$. The cells proliferate to form the dental placode, which continues through the bud, cap and bell stages induced by signalling interactions between the epithelium and the mesenchyme. In the bell stage, cells differentiate into ameloblasts and odontoblasts followed by matrix secretion and mineralisation of the crown ${ }^{9}$. Root development which is tightly genetically controlled, extends from the Hertwigs's Epithelial Root Sheath (HERS). The HERS is formed from the cervical loop which consists of the inner and outer enamel epithelium at the neck ring of the dental papilla ${ }^{10}$. What exactly is the decisive component triggering the HERS to initiate root formation is not well known, but Sakano et al recently showed that the outer enamel epithelium cells had a higher proliferative and migratory activity than the inner enamel epithelial cells before forming the HERS ${ }^{11}$. 
The HERS cells are then thought to initiate odontoblast differentiation from mesenchymal cells and to determine root size and shape ${ }^{12,13}$. Other authors have reported a novel cell population, the apical odontoblast, as being responsible for the initiation of root formation ${ }^{14}$. These cells lie apically to the HERS after crown formation and they are dependent upon the activation of $\beta$-catenin ${ }^{14,15}$. It is clear that disturbances in this finely tuned process might affect root development.

In this paper, we aim to describe six cases of root malformations that were referred to the Department of Paediatric Dentistry at the University of Oslo and to the Oral Health Centre of Expertise in Mid-Norway recently. The condition was unfamiliar and differential diagnoses included a progressive resorptive process or a developmental defect. After observing the cases over time and performing histological and microCT examinations, we now know that all these cases demonstrate developmental disturbances of root development. There are no signs that indicate a resorptive lesion in any of the cases. We also hope to give a schematic guide to identify the condition in children with a known serious medical condition during the first years of life, and a guide to describing and reporting the condition when discovered. 


\section{Methods}

\section{Patients}

These patients were all referred to our department or to the Oral Health Centre of Expertise in MidNorway from the public dental health service or from private general dentists. The patients' teeth and oral cavities were examined clinically and radiographically. Clinical images were taken at the first visit with a Canon EOS60D camera with Canon MR-14EX Ring Flash and a 100-mm Canon Macro Lens EF (Tokyo, Japan). Characterizations of the patients are summarized in Table 1. Written informed consent was given by the patients' parents. Teeth are designated with the FDI (World Dental Federation) notation (ISO 3950).

\section{Histology}

The extracted molar 46 from patient 1 was embedded in resin (Technovit 7100) without decalcification after rehydration and then sectioned in the bucco-lingual direction. Images were obtained with a Canon EOS60D camera (Tokyo, Japan) with Canon MR-14EX Ring Flash and a 100-mm Canon Macro Lens EF and a Zeiss Discovery.V8 SteREO stereomicroscope with an Axiocam 105 color camera (Carl Zeiss Meditec AG, Jena, Germany). Ten $\mu$ m sections were made and mounted on glass slides. For visualisation, hematoxylin was applied to the undecalcified surface for three minutes, slides were washed in distilled water and air dried, then eosin was applied for three minutes before washing and drying. The sections were evaluated under a Leica DMRBE microscope (Leica Mikrosysteme Vertrieb GmbH, Mikroskopie und Histologie, Wetzlar, Germany) and images were taken with an Olympus DP50 camera (Olympus Corporation, Tokyo, Japan) fitted to the microscope.

\section{MicroCT}

Molar 36 from patient 1 was scanned in a SkyScan 1172 (SkyScan, Aartselaar, Belgium) $\mu$ CT with an image pixel size of $11.95 \mu \mathrm{m}$ and an image resolution of $2000 \times 1048$ pixels. The $360^{\circ}$ scans were 
obtained at $100 \mathrm{kV}, 100 \mu \mathrm{A}$ at $0.4^{\circ}$ rotation steps with an additional aluminium and copper filter (Al $1 \mathrm{~mm}$ and $\mathrm{Cu} 0.05 \mathrm{~mm}$ ) in the beam path.

The scans were reconstructed using the standard SkyScan reconstruction software (NRecon,

v1.6.10.1). The following parameters were applied: smoothing of 2 pixels, beam hardening correction of $25 \%$, ring artefact reduction of 12 and an output attenuation coefficient range of $0.00-0.1$. 


\section{Results}

\section{Cases}

A summary of the patient cases is given in Table 1.

\section{Patient 1}

This 8-year-old female was referred to the university clinic because of painful lower first permanent molars on both sides with abnormal roots. The pain had been intermittent for 2-3 months, gradually worsening and occasionally preventing her from sleeping. The clinical and radiographic examination revealed narrow and underdeveloped roots, obstructed pulp cavities and bone destruction down to the furcation areas (Figure 1). The right mandibular first molar demonstrated an apical radiolucency and a radiolucent area along the mesial surface of the mesial root all the way from the cervical to the apical margin (Figure 1). There were no marginal pockets deeper than $3 \mathrm{~mm}$ to be probed at the time of examination, but the referring dentist had discovered marginal pockets deeper than $3 \mathrm{~mm}$ on both teeth earlier. The upper first permanent molars also showed underdeveloped roots. No bone loss was detected on the radiographs of the upper jaw. The two lower first primary molars showed taurodontism. The patient was healthy, but had a medical record of brain blood clot at birth which was treated with an anticlotting agent and antibiotics for four weeks under hospitalization. She had experienced occasional epileptic seizures up to the age of seven. These were treated with midazolam. The most painful tooth, the lower left mandibular molar (36) was extracted the same day, and 46 the following week. These teeth were examined further (Figure 2 and 3). This gave pain relief, and complete healing occurred.

\section{Patient 2}


This female was 11 years old at referral due to swelling and suppuration from 46 . Radiographs revealed missing roots in 36 and 46 , accompanied by a $12 \times 13 \mathrm{~mm}$ radiolucency occupying the whole area where the root of 46 would have been expected (Figure 4). The roots of 16 and 26 were malformed and short and pulp chamber obliteration was seen. The upper central incisors showed constrictions in the cervical third of the crown while the roots were normal. A white, demarcated enamel opacity could be seen on the upper left first molar. Radiographic and clinical images are shown in Figure 4. An abdominal tumour was detected by foetal ultrasound examination, and surgically removed at three months of age.

\section{Patient 3}

This 9 year old male was referred for evaluation because of incomplete root development in the second primary and first permanent molars, in addition to hypersensitivity to cold probably because of MIH on all first permanent molars. The clinical and radiographical examination revealed very tiny wedge-shaped roots on all four first permanent molars and roots that were less than $1 \mathrm{~mm}$ long on the second primary molars (Figure $5 \mathrm{~A}$ ). The upper left primary molar was already missing, and mesial drift of the permanent molar 26 had occurred. In addition, he had MIH affecting the maxillary central incisors and first permanent molars (Figure $6 \mathrm{~A}, \mathrm{~B}, \mathrm{C}$ ). The male was born full-term, but 10 days after birth he experienced multiple brain abscesses. He was then hospitalized and underwent surgery and antibiotic therapy several times. He also experienced episodes of high fever, and was hospitalized several times until he was 18 months old. His mother had used a selective serotonin reuptake inhibitor during pregnancy.

\section{Patient 4}

This nine year old female was referred because of atypical roots on her molars and maxillary incisors. She had no symptoms from the teeth and was otherwise healthy. Radiographs showed narrow, short 
roots with partly obliterated pulp chambers and hypercalcified and malformed cervical area on all first permanent molars (Figure 5 B and Figure 6 D, E, F). The upper central incisors had constrictions in the cervical area, but the roots appeared normal. The female was born full-term by acute caesarean section due to placental abruption. She had a cavernous haemangioma in her left cheek, and was hospitalized the first 11 days after birth.

\section{Patient 5}

This 12 year old male was referred after repeated episodes of fever, pain and swelling from 46 . Radiographs showed a cervical constriction and obliterated pulp chamber but long, straight roots on both 36 and 46 . There were apical radiolucencies on the mesial roots of both teeth. Clinical examination revealed an abscess with a draining sinus mesial to the roots of 46 . The upper first molars showed the same cervical malformation, but their roots were shorter and narrower. His incisors were normal (Figure $5 \mathrm{C}$ and Figure $6 \mathrm{G}, \mathrm{H}, \mathrm{I}$ ). Due to complicated labour, the male was born by vacuum-assisted delivery. He also had a tight nuchal cord which caused asphyxia. Intracranial haemorrhage was present and he had cerebral palsy. He presently uses methylphenidate.

\section{Patient 6}

This 11 year old female was also referred due to pain and swelling in 36 . Radiographs revealed obliterated pulp chambers, missing distal roots and thin mesial roots in 36 and 46 . The maxillary first molars had partly obliterated pulp chambers and somewhat narrow roots. The incisors were normal (Figure $5 \mathrm{D}$ and Figure $6 \mathrm{~J}, \mathrm{~K}, \mathrm{~L}$ ). Five days after birth, she had experienced seizures and an ischemic stroke on the left side of the brain.

\section{MicroCT evaluation of an extracted molar}


Two painful molars were extracted from patient 1. A panoramic radiograph obtained at the first visit is shown in Figure 1. The microCT scan of tooth 36 revealed a partly obliterated pulp chamber connected to one root canal only (Figure 2). The coronal dentin and enamel were of normal appearance. Cervically to the pulp chamber, a layer of dentin with normal density was seen, and then a denser layer appeared. The hyper-calcified (denser) layer corresponded to a cervical enamel constriction. The three dimensional reconstruction showed numerous root canals not connected to the pulp chamber.

\section{Morphological appearance of extracted molars}

Clinical images of molar 36 from patient 1 were obtained right after extraction (Figure $3 \mathrm{~A}, \mathrm{~B}$ ). This was the same tooth that was evaluated with microCT (Figure 2). The images showed a long distal root and short, bent irregular mesial roots (Figure $3 \mathrm{~A}, \mathrm{~B}$ ). A cervical constriction and a more prominent area apical to the constriction were seen on the buccal side (Figure $3 \mathrm{~A}$, arrow). Between the short mesial roots, a hyperplastic soft tissue nodule was seen (Figure $3 \mathrm{~B}$, arrow). The extracted tooth 46 from the same patient was embedded in resin and cut in the bucco-lingual direction (Figure 3 C-F). This section showed a narrow pulp chamber with small calcified rods extending from the walls (Figure 3C, black arrow and inserted enlargement). As seen in the hematoxylin-eosin (H\&E)-stained sections, the floor of the pulp chamber consisted of a layer of normal looking dentin (Figure 3D, black arrow). Cervical to the normal dentin layer an irregular dentin layer appeared (Figure 3D, white arrow), similar to what was seen on the microCT scan. The H\&E-stained sections showed that this dentin layer consisted of two layers; one irregular (Figure 3E, black arrow) and one of globular appearance (Figure 3E, white arrow). The same layers can be seen from a different view of the same tooth (Figure 3F). The hyper-calcified dentin layer seemed to obturate the connection between the pulp chamber and the root canals (Figure 3C, white arrow). 


\section{Discussion}

The prevalence of this newly described condition is so far unknown as the only descriptions are the case reports discussed here ${ }^{2-7}$. However, it is likely that this is not a new condition, and the fact that we are now aware of it, helps us detect the condition and treat affected individuals appropriately. Probably, many of these teeth would have been extracted once they became symptomatic, or demonstrated periodontal or apical infection, possibly in combination with acute pulpitis. The patient could have been misdiagnosed with a resorptive process. On the other hand, some of the systemic conditions these children have experienced during their first years of life might have been so treatment intensive that they would not have survived a couple of decades ago. If so, one could speculate that the incidence of this condition could increase with improved perinatal health care.

There seems to be an association between the environmental stressors these children experienced early in life and the root molar malformations and incisor constrictions. This was also suggested by Wright et al $^{6}$ and Luder ${ }^{16}$. However, the aetiology of the condition remains unclear. Initial mineralisation of first permanent molars starts around birth. The crown is fully mineralised at the age of three, then the furcation forms, followed by root development ${ }^{9}$. The timing of disease onset (the first year of life) and root development of first permanent molars (around the age of three years) do not coincide and this remains a puzzle. One explanation might be that the HERS is already formed long before root development starts; it awaits signals from the mesenchymal cells to differentiate and later disintegrate during root formation. Early disturbances in the epithelial-mesenchymal crosstalk might affect the ability of these cells to differentiate properly into odontoblasts. Both environmental and genetic conditions may impair root development. A study in mice showed that radiation of 10 and 20 Grey before the onset of root development disrupted the HERS and gave root malformation ${ }^{17}$. A genetic study using Nuclear factor $\mathrm{I}-\mathrm{C}$ (Nfic) knockout mice demonstrated 
disturbances in root patterning leading to taurodontism in these mice ${ }^{18}$. The study also demonstrated that mesenchymal proliferation was impaired causing short roots in the mutant mice. It was suggested by Wright et al ${ }^{6}$ that neurologic conditions could be an aetiological factor, since many of the children in their study had conditions related to neurological damage. This does not seem to be the case in our study. Even though most of our patients suffered from conditions in the brain or head, these conditions seemed rather to be of vascular nature, such as cranial haemorrhage or infarct. Several studies report the contribution of neural crest cells to dental mesenchyme, and thereby suggest a connection between brain tissue and teeth ${ }^{19,20}$. In the present cases, a relationship between brain damage and developmental disorders of the teeth would only be speculation. However, the patients might have a genetic predisposition favouring damage to both teeth and brain tissue.

In addition to root malformation with pulpal obliteration and cervical constriction, one of the patients had taurodontic first mandibular primary molars (Figure 1). The aetiology of taurodontism may be both genetic and environmental and can be associated with syndromes including other oral aberrations ${ }^{16,21,22}$. A taurodont arises when the HERS fails to start the formation of the furcation bridges ${ }^{16}$, but this seems just to be a delay, since a normal furcation will be formed later on. This is in contrast to what is seen in the MIM teeth where the furcation is malformed at its normal location. We cannot exclude a possible common genetic cause or predisposition for the two different root anomalies seen in patient 1 , but the two anomalies may be appearing simultaneously by coincidence. Even though most of the MIM teeth looked normal in the mouth, there were exceptions. The affected incisors of patients 2 and 4 had interproximal constrictions (Figure 4 and Figure 6 D). Patient 2 showed a demarcated opacity on the upper left first molar (Figure 4), while patient 3 had demarcated opacities both on his first permanent molars and his incisors (Figure A, B, C), consistent with an $\mathrm{MIH}$ diagnosis ${ }^{23}$. Given that illness during the first three years might be an aetiological factor of $\mathrm{MIH}$ it is not surprising that some of these patients show signs of $\mathrm{MIH}^{24}$. 
The macroscopic, microscopic and radiographic appearance of the teeth examined in this study (Figures 1-6) were comparable with the teeth described by Witt et al and Lee et al ${ }^{2,4}$, making us confident that we are describing the same condition. Further studies are needed to clarify the aetiology and mechanisms of development. We suggest a diagnosis chart with check points for the condition for use when treating children with a known medical history of a serious medical condition, particularly related to the head and neck region or the brain early in life (Figure 7). We also suggest a registration chart to use when root malformations are discovered (Figure 8). By actively looking for the condition, and encouraging clinicians also to do so, we hope to learn about the incidence and the prevalence of the condition.

The children with MIM must be followed closely by the dental team. In cases of severe malformation with poor long-term prognoses, extractions should be planned to minimize the need for painful and complicated treatment later. Many of these cases would probably benefit from extraction, allowing the healthy second permanent molar to replace the first. Cooperation with an orthodontist in the treatment planning would be wise, and advice on early extraction of first permanent molar can be found in the British clinical guidelines for the extraction of first permanent molars ${ }^{25}$.

\section{Acknowledgement}

We thank for the skilful help from Jonas Wengenroth and Shabhaz Yousefi at Clinical Oral Research Laboratory for the microCT scans and laboratory work. 


\section{References}

1 Jalevik, B. Prevalence and Diagnosis of Molar-Incisor- Hypomineralisation (MIH): A systematic review. Eur. Arch. Paediatr. Dent. 11, 59-64 (2010).

2 Witt, C. V., Hirt, T., Rutz, G. \& Luder, H. U. Root malformation associated with a cervical mineralized diaphragm--a distinct form of tooth abnormality? Oral Surg Oral Med Oral Pathol Oral Radiol 117, e311-319, doi:10.1016/j.0ooo.2013.06.030 (2014). Lee, H. S. et al. A new type of dental anomaly: molar-incisor malformation (MIM). Oral Surg Oral Med Oral Pathol Oral Radiol 118, 101-109.e103, doi:10.1016/j.00oo.2014.03.014 (2014). Lee, H. S. et al. Microscopic analysis of molar--incisor malformation. Oral Surg Oral Med Oral Pathol Oral Radiol 119, 544-552, doi:10.1016/j.00oo.2014.10.013 (2015).

Yue, W. \& Kim, E. Nonsurgical Endodontic Management of a Molar-Incisor Malformationaffected Mandibular First Molar: A Case Report. J. Endod. 42, 664-668, doi:10.1016/j.joen.2015.11.004 (2016). Wright, J. T. et al. Molar root-incisor malformation: considerations of diverse developmental and etiologic factors. Oral Surg Oral Med Oral Pathol Oral Radiol 121, 164-172, doi:10.1016/j.0ooo.2015.08.024 (2016). McCreedy, C., Robbins, H., Newell, A. \& Mallya, S. M. Molar-incisor Malformation: Two Cases of a Newly Described Dental Anomaly. J Dent Child (Chic) 83, 33-37 (2016). Bei, M. Molecular genetics of tooth development. Curr. Opin. Genet. Dev. 19, 504-510, doi:10.1016/j.gde.2009.09.002 (2009).

9 Koch, G., Thesleff, I. \& Kreiborg, S. in Pediatric Dentistry- A clinical Approach Ch. 4, 28-39 (Wiley Blackwell, 2017).

10 Huang, X. F. \& Chai, Y. Molecular regulatory mechanism of tooth root development. Int J Oral Sci 4, 177-181, doi:10.1038/ijos.2012.61 (2012).

11 Sakano, M. et al. Cell dynamics in cervical loop epithelium during transition from crown to root: implications for Hertwig's epithelial root sheath formation. J. Periodontal Res. 48, 262267, doi:10.1111/jre.12003 (2013).

12 Ten Cate, A. R. The role of epithelium in the development, structure and function of the tissues of tooth support. Oral Dis. 2, 55-62 (1996).

13 Thomas, H. F. \& Kollar, E. J. Differentiation of odontoblasts in grafted recombinants of murine epithelial root sheath and dental mesenchyme. Arch. Oral Biol. 34, 27-35 (1989). Bae, C. H., Kim, T. H., Chu, J. Y. \& Cho, E. S. New population of odontoblasts responsible for tooth root formation. Gene Expr Patterns 13, 197-202, doi:10.1016/j.gep.2013.04.001 (2013). $\mathrm{Kim}, \mathrm{T}$. H. et al. beta-catenin is required in odontoblasts for tooth root formation. J. Dent. Res. 92, 215-221, doi:10.1177/0022034512470137 (2013).

16 Luder, H. U. Malformations of the tooth root in humans. Front. Physiol. 6, 307, doi:10.3389/fphys.2015.00307 (2015).

17 Ide, Y., Nakahara, T., Nasu, M. \& Ishikawa, H. Cell dynamics in Hertwig's epithelial root sheath and surrounding mesenchyme in mice irradiated to the head. Oral Dis. 21, 232-239, doi:10.1111/odi.12253 (2015). growth. Anat. Cell Biol. 48, 188-194, doi:10.5115/acb.2015.48.3.188 (2015). Miletich, I. \& Sharpe, P. T. Neural crest contribution to mammalian tooth formation. Birth Defects Res C Embryo Today 72, 200-212, doi:10.1002/bdrc.20012 (2004).

20 Chai, Y. et al. Fate of the mammalian cranial neural crest during tooth and mandibular morphogenesis. Development 127, 1671-1679 (2000). 
21 Haskova, J. E., Gill, D. S., Figueiredo, J. A., Tredwin, C. J. \& Naini, F. B. Taurodontism--a review. Dent. Update 36, 235-236, 239-240, 243 (2009).

22 Dineshshankar, J. et al. Taurodontism. J. Pharm. Bioallied Sci. 6, S13-15, doi:10.4103/09757406.137252 (2014).

23 Weerheijm, K. L. et al. Judgement criteria for molar incisor hypomineralisation (MIH) in epidemiologic studies: a summary of the European meeting on MIH held in Athens, 2003. Eur. J. Paediatr. Dent. 4, 110-113 (2003).

24 Lygidakis, N. A. et al. Best Clinical Practice Guidance for clinicians dealing with children presenting with Molar-Incisor-Hypomineralisation (MIH): An EAPD Policy Document. Eur. Arch. Paediatr. Dent. 11, 75-81 (2010).

25 Cobourne, M. T., Williams, A. \& Harrison, M. National clinical guidelines for the extraction of first permanent molars in children. Br. Dent. J. 217, 643-648, doi:10.1038/sj.bdj.2014.1053 (2014). 


\section{Figure legends}

\section{Figure 1:}

Panoramic radiograph of patient 1 obtained at the first visit. All four first permanent molars demonstrate malformed roots. Tooth 36 has a radiolucent area in the cervical/mesial part and tooth 46 has a radiolucent area along the entire mesial root and periapical to the distal root. Both 36 and 46 have narrow pulp chambers. First primary lower molars on both sides are taurodonts and are marked with a T.

\section{Figure 2:}

MicroCT scan of tooth 36 from patient 1. The scan shows two vertical (A, C) and one horizontal (B) sections in addition to a three dimensional reconstruction (D). The coloured lines indicate the direction of the sections. A: Vertical section. Cervical constriction (arrowhead). Cervical to the pulp chamber is a normal dentin layer (White d) and a more densely calcified area (arrow). e=enamel B: Horizontal section through the tooth at the level of the red line in $A$ and $C$ by the floor of the pulp chamber. C: The pulp chamber $(p)$ is partially obstructed, connected to one of the root canals only. The more densely calcified area is seen cervical to the pulp floor (arrow). D: A three dimensional reconstruction of the scans from the whole tooth. Numerous oddly formed roots and root canals are seen.

\section{Figure 3:}

Photographs of a complete and sectioned first permanent molar from patient 1. A: Buccal view of tooth 36 (the same tooth as seen in figure 2) immediately after extraction. At the cervical $1 / 4$ of the buccal surface there is a prominence of enamel. The arrow points to this bucco-cervical prominence. B: Mesial view of tooth 36. Hyperplastic tissue nodule is seen between the short mesial roots (arrow) C: Bucco-lingual section of tooth 46 embedded in resin. A narrow pulp chamber (black arrow) and an irregular dentin layer (white arrow) is seen. The insert is a larger magnification of the area in the 
black box. D: HE-stained section showing a narrow pulp chamber, a layer of normal dentin (black arrow) and irregular dentin (white arrow). E: Larger magnification of the irregular dentin. One layer of irregular (black arrow) and one of globular appearance (white arrow) can be seen. F: Section from the opposite side of the pulp chamber in $D$, showing a narrow pulp chamber, a layer of normal dentin and irregular dentin.

The H\&E-stained sections were examined under a Nikon microscope fitted with a 10X (D, F) and 40X magnification lens (E).

\section{Figure 4:}

Panoramic radiograph and clinical photographs of patient 2. The radiograph shows narrow, wedgeshaped roots on the upper permanent first molars, and missing roots on the lower first permanent molars. Cervical crown constrictions can be seen on the upper permanent incisors. Clinical images show normal appearance of teeth in the lower jaw (left), a white demarcated enamel opacity was seen on upper left molar (arrow, middle image) and cervical crown constrictions on upper incisors (right).

\section{Figure 5:}

Panoramic radiographs of patients 3, 4, 5 and 6.

A: Patient 3. Small wedge-shaped roots on all four first permanent molars. Lower second primary molars have less than $1 \mathrm{~mm}$ long roots. Upper left second primary molar is missing.

B: Patient 4. Small, malformed roots on all four first permanent molars. Cervical constrictions can be seen on upper permanent incisors.

C: Patient 5. Small, wedge-shaped roots on upper first permanent molars. Malformed cervical area , long roots with apical radiolucency can be seen on both lower first permanent molars. 
D: Patient 6. Upper first permanent molars have narrow pulp chambers, but normal root morphology. Lower first permanent molars show cervical malformation and missing distal roots.

\section{Figure 6:}

Clinical images of patients 3, 4, 5 and 6 .
A, D, G, J: Frontal view of the teeth
$B, E, H, K$ : Occlusal view of the upper jaw.
C, F, I, L: Occlusal view of the lower jaw.

A: Arrow shows white enamel opacity on patient 3.

B, C: Arrows show brown and white enamel opacities on patient 3.

D: Arrow shows cervical constriction on patient 4.

\section{Figure 7:}

Check point chart for diagnostic use. The chart lists useful items to assist in discovering the condition when treating children with known serious medical conditions early in life.

Figure 8:

Suggested workflow to apply on discovering a tooth with missing or malformed roots. 
Table I. Summary of patients included in the study

\begin{tabular}{clllll} 
Patient \# & Medical history & Clinical characterization & Affected teeth & Age & Sex \\
\hline 1. & $\begin{array}{l}\text { Born with brain blood } \\
\text { clot, antibiotic and }\end{array}$ & Short hypoplastic and twisted & 16, 26, 36, 46 & 8 & $\mathrm{~F}$ \\
anticlotting agents for & roots of lower permanent first & & & \\
four weeks. Epileptic & malformation of buccocervical & & \\
seizures until the age & part of crown of 36. Pain and & & \\
& $\begin{array}{l}\text { of 7, treated with } \\
\text { midazolam. }\end{array}$ & swelling. Underdeveloped roots & & \\
& & of upper molars, no infection. & & \\
& & Taurodontic 74 and 84. & &
\end{tabular}

2. Abdominal tumour

Swelling and pus from 46 with

$16,26,36,46$,

$11 \mathrm{~F}$ detected by foetal ultrasound corresponding large 11,21 examination, surgically radiolucency. Missing roots in removed at 3 months 36 and 46, malformed roots 16 , of age.

26. Cervical constrictions in upper central incisors. White demarcated opacity on upper first molar.

3. Hospitalized several times up to 1.5 years of age due to brain Tiny wedge-shaped roots on first permanent molars, no $16,26,36,46, \quad 9$ $55,75,85$ abscesses and high roots on three second primary molars, one is missing. fever. Hydrocephalus, Enamel hypomineralisation three ventricle shunts. $(\mathrm{MIH})$ on molars and incisors.

4. Acute caesarean section, zygomatic cavernous haemangioma.

5. Difficult delivery: tight nuchal cord, birth asphyxia, intracranial haemorrhage. Cerebral palsy.

6. Seizures and ischemic stroke (left side of brain) 5 days after birth.
Deformed and narrow roots on first permanent molars. Cervical constrictions on upper incisors.

Fever, pain, swelling from 46. Root malformations and cervical constrictions in first permanent molars.

Pain and abscess in 36. Missing $16,26,36,46$ 11

$16,26,36,46, \quad 9 \quad \mathrm{~F}$ 11,21

$16,26,36,46$ 12 M distal and narrow mesial roots in 36 and 46, narrow roots in 16 and 26 .

Dental notations in ISO system according to WHO, age in years. M=male F=Female 


\section{Figure 1}

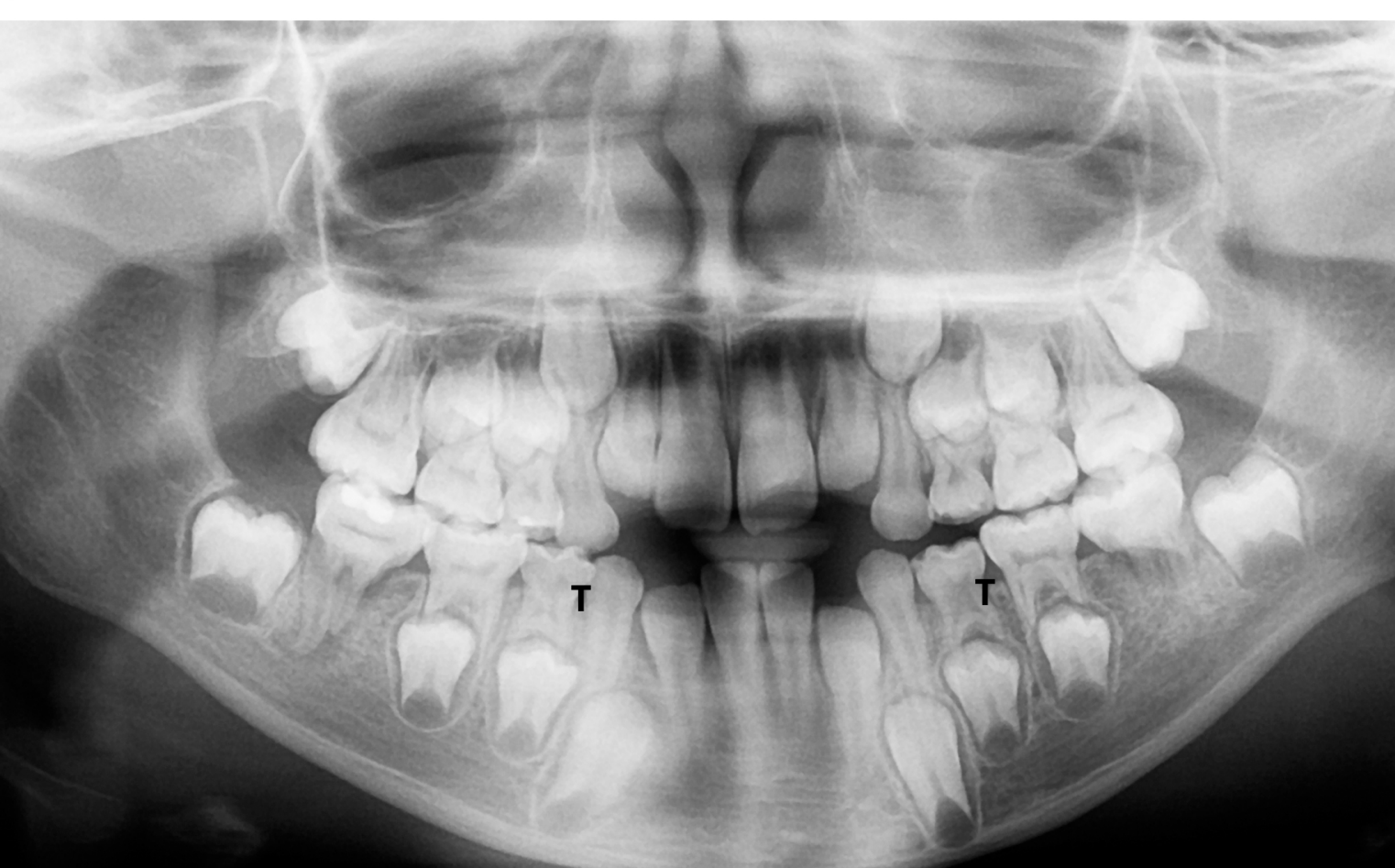


Figure 2
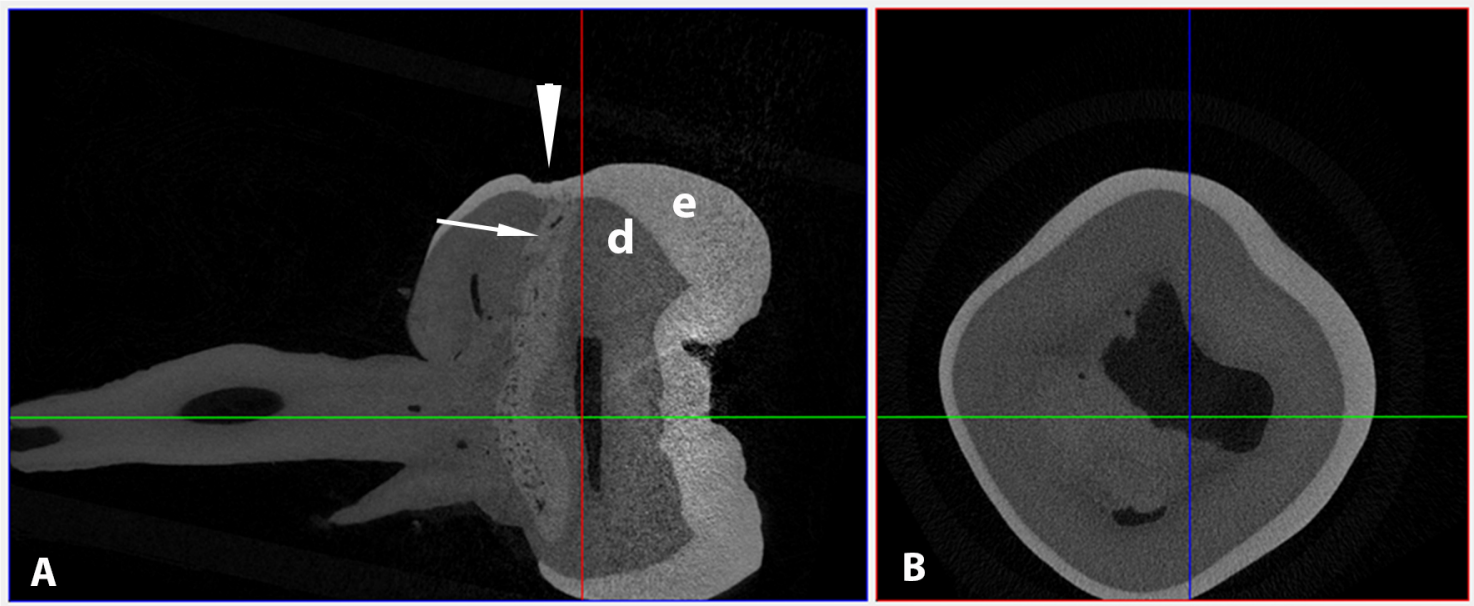

D
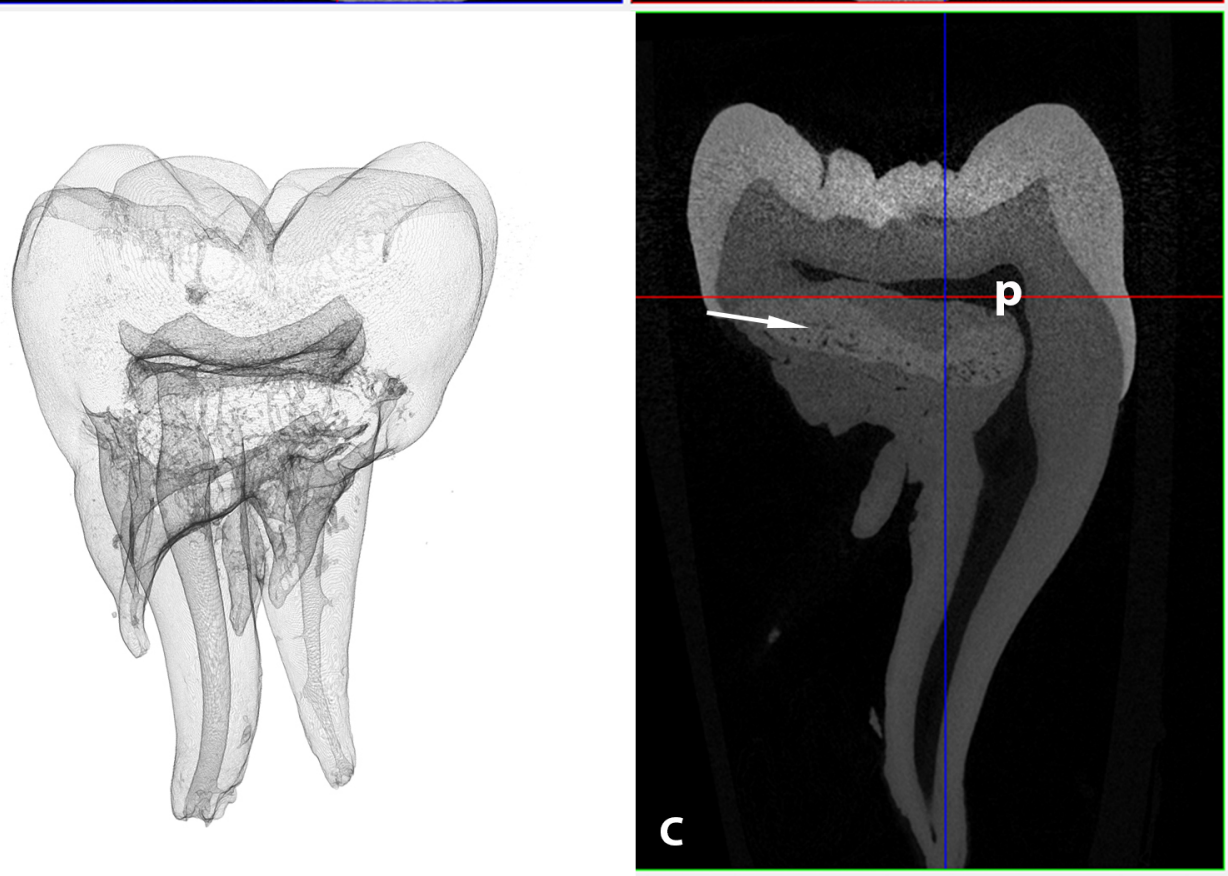


\section{Figure 3}
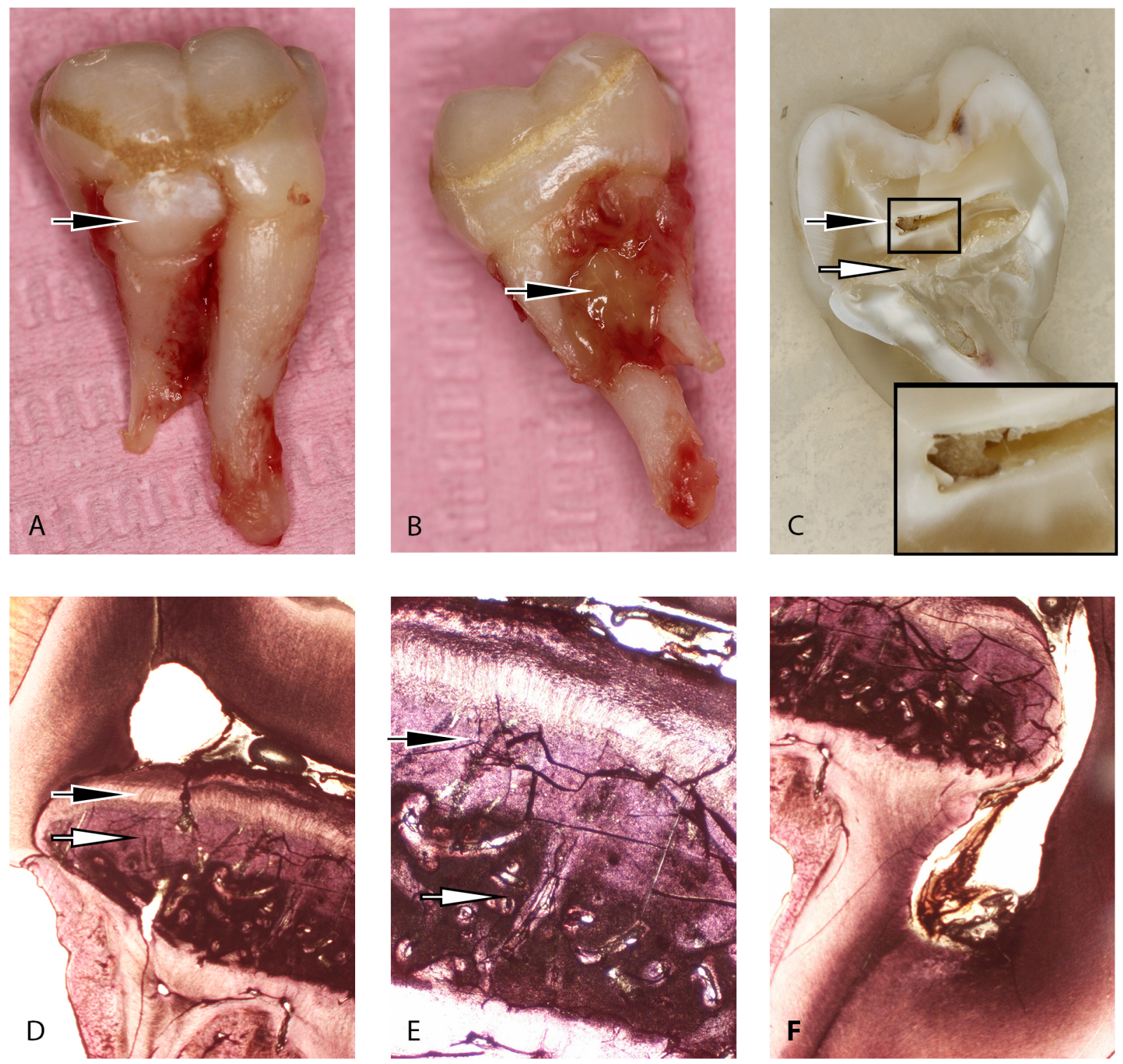

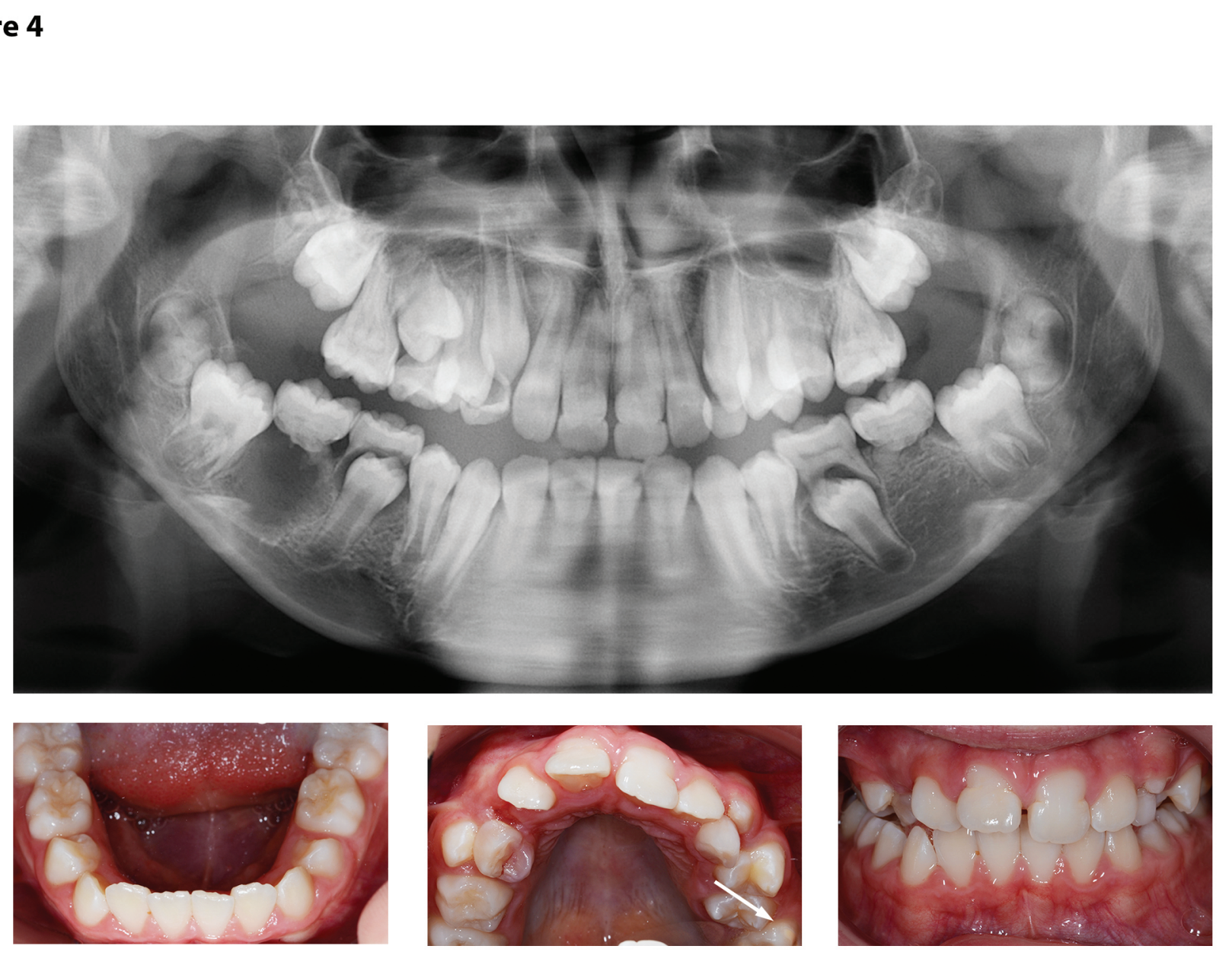

\section{Figure 4}
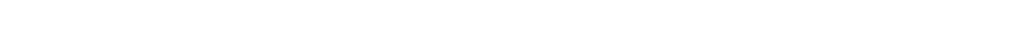

.

.
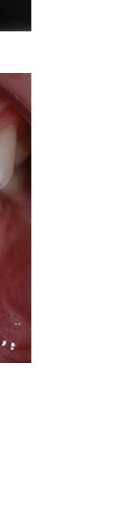

rar

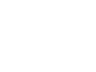




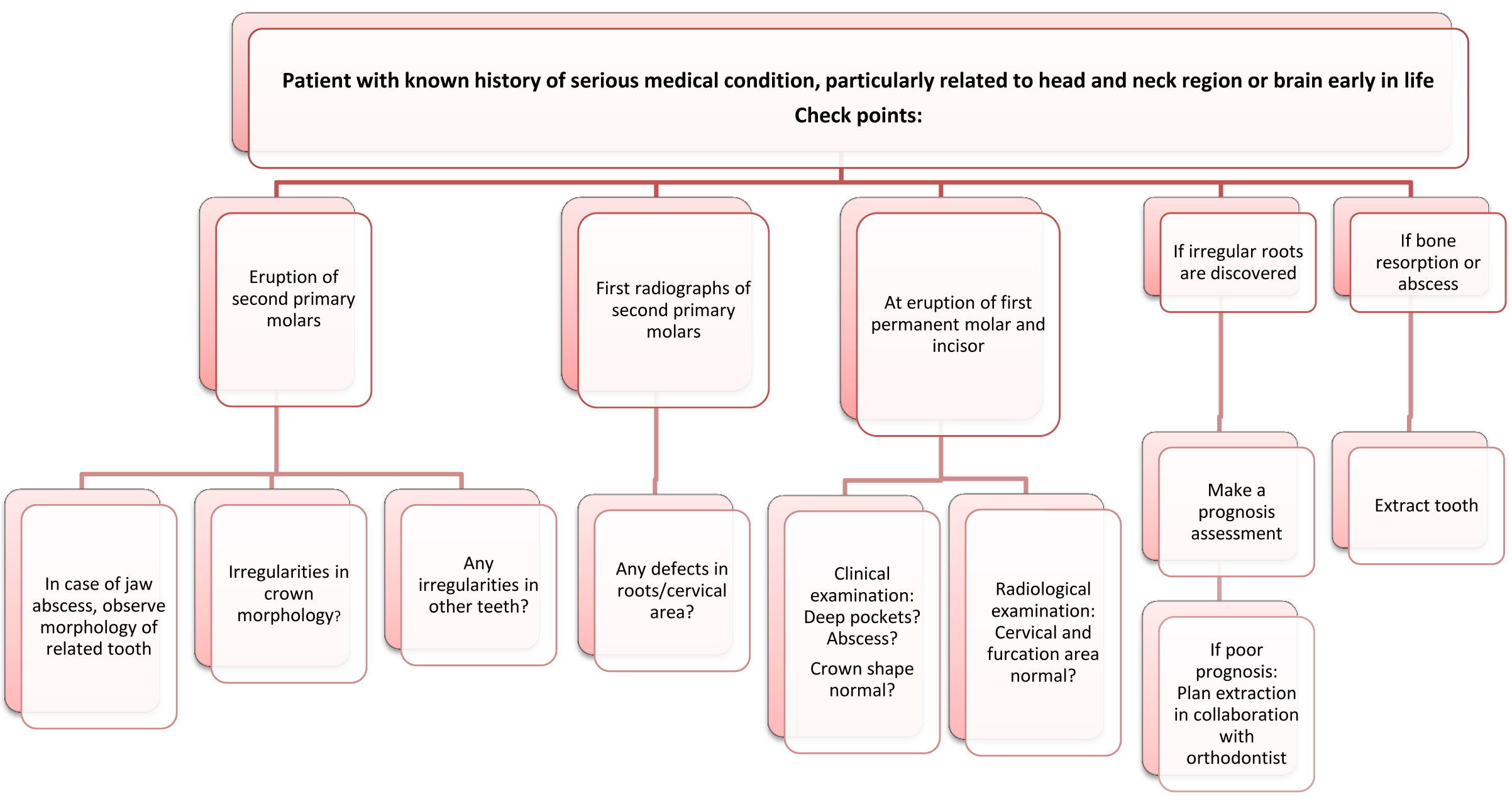


Figure 8

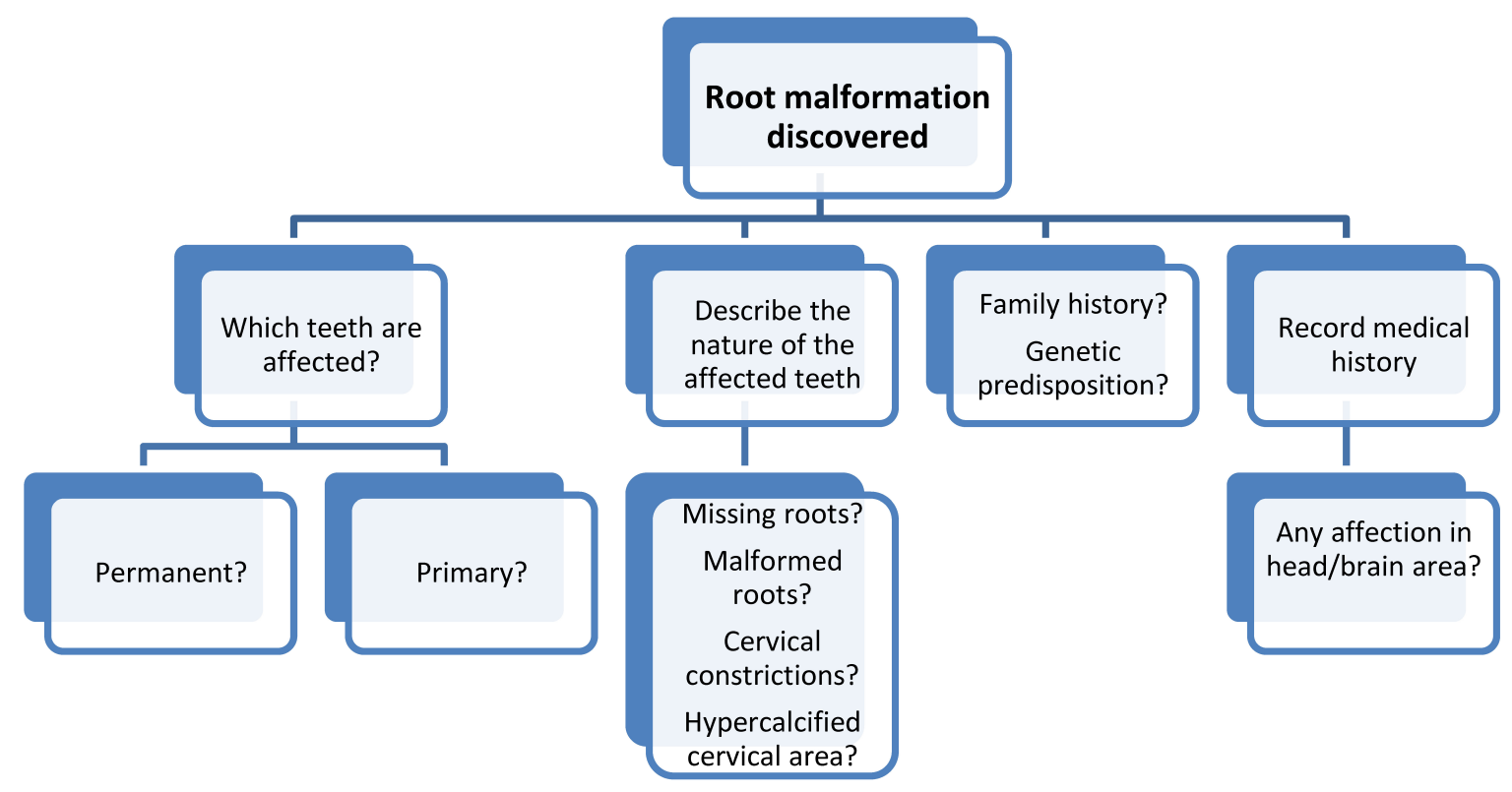

